\title{
Disorder-Induced Resistive Anomaly Near Ferromagnetic Phase Transitions
}

\author{
Carsten Timm, ${ }^{1}$ M. E. Raikh, ${ }^{2}$ and Felix von Oppen ${ }^{1}$ \\ ${ }^{1}$ Institut für Theoretische Physik, Freie Universität Berlin, Arnimallee 14, 14195 Berlin, Germany \\ ${ }^{2}$ Department of Physics, University of Utah, Salt Lake City, UT 84112
}

(Dated: August 27, 2004)

\begin{abstract}
We show that the resistivity $\rho(T)$ of disordered ferromagnets near, and above, the Curie temperature $T_{c}$ generically exhibits a stronger anomaly than the scaling-based Fisher-Langer prediction. Treating transport beyond the Boltzmann description, we find that within mean-field theory, $d \rho / d T$ exhibits a $\left|T-T_{c}\right|^{-1 / 2}$-singularity near $T_{c}$. Our results, being solely due to impurities, are relevant to ferromagnets with low $T_{c}$, such as $\mathrm{SrRuO}_{3}$ or diluted magnetic semiconductors, whose mobility near $T_{c}$ is limited by disorder.

PACS numbers: 72.10.Fk, 72.20. My, 75.50.Cc, 75.50.Pp
\end{abstract}

Introduction.-It was first observed by Gerlach [1] that the resistivity of itinerant ferromagnets exhibits an anomalous temperature dependence in the vicinity of the Curie temperature $T_{c}$. This feature was later reproduced with much higher experimental accuracy by Craig et al. 2] as well as others [3]. Dating back to the seminal works of de Gennes and Friedel [4] as well as Fisher and Langer [5], this resistive anomaly is conventionally explained in terms of coherent scattering of carriers by large blocks of spins whose size is determined by the magnetic correlation length $\xi(T)$. As the temperature approaches $T_{c}$, $\xi(T)$ diverges, making the scattering more efficient.

de Gennes and Friedel [4] studied the resistive anomaly of ferromagnets within mean-field (MF) theory. They argue that due to critical slowing down, spin fluctuations can be treated as effectively static. The ensuing result for the coherent transport scattering rate from spin fluctuations above $T_{c}$ is succinctly summarized by [4]

$$
\frac{\tau_{0}}{\tau}=\frac{1}{4} \int d x \frac{x^{3}}{t+\left(k_{F} a x\right)^{2}} .
$$

Here, the scattering rate is normalized to the rate $1 / \tau_{0}$ for incoherent scattering. The denominator of the integrand arises from the conventional MF (Ornstein-Zernike) correlator for spin fluctuations, with $t=\left(T-T_{c}\right) / T_{c}$ denoting the reduced temperature. $a$ is a microscopic length of the order of the lattice constant. The integration variable $x$ denotes the transferred momentum in units of the Fermi wave vector $k_{F}$. The numerator incorporates the usual factor $1-\cos \theta$ (with $\theta$ the scattering angle) in the transport scattering rate. The integral in Eq. (11) yields $\tau_{0} / \tau(t)=\tau_{0} / \tau(0)+\left(1 / 8\left(k_{F} a\right)^{4}\right) t \ln t$, implying a singularity of the resistivity of the form $\rho(T)=\rho_{0}-b t \ln (1 / t)$ with $b>0$ when approaching $T_{c}$ from above.

Fisher and Langer [5] noticed that this singularity emerges from the lower limit of the integral in Eq. (II) while the body of the integrand is dominated by large wave vectors. Within MF theory, the large-wave-vector behavior of the spin-spin correlator is non-singular. The central assertion of Ref. [5] is that there exists a singular contribution to this correlator at large wave vectors when going beyond the MF approximation, with the singularity governed by anomalous dimensions. Fisher and Langer conclude that while below $T_{c}$, the predictions of Ref. [4] are essentially correct, there is no singularity within $M F$ approximation when approaching $T_{c}$ from above.

This conclusion rests on their important physical observation that Eq. (11) becomes inapplicable for $t \ll$ $(\ell / a)^{2}$, or equivalently, when the correlation length $\xi(T)$ exceeds the mean free path $\ell$ (due to phonon or impurity scattering). The reason is that the carriers can be viewed as plane waves only over distances shorter than $\ell$ and thus, they are no longer susceptible to the order in the spin configuration beyond $\ell$.

Later, the ideas of Refs. [4, 5] were extended to include realistic features of ferromagnets [6] and to the critical behavior of other quantities such as the spin-flip scattering rate $7,[8,9]$. The effect of a finite mean free path on the resistivity was studied by a number of approaches, ranging from replacing the $\delta$-function in the golden rule by a Lorentzian [8] to smearing the Ornstein-Zernike correlator in order to eliminate the pole 10].

All of these approaches are based on the Boltzmannequation formalism. The prime message of this paper is that in the small- $t$ limit, when the correlation length $\xi(T)$ exceeds the mean free path $\ell$, the Boltzmann approach fails. The reason for this is that for $\xi(T) \gg \ell$, the smooth variations of the magnetization (on the scale $\xi(T))$ are "explored" by diffusing carriers. By contrast, the Boltzmann approach prescribes to treat scattering from both short-range impurities and the smooth variations of the magnetization on equal footing, i.e., to add their partial scattering rates.

The consequences of going beyond the Boltzmann approach are drastic. In fact, as demonstrated below, instead of smearing the resistive anomaly, impurities cause a much stronger singularity, even within MF theory. Quantitatively, we find $d^{2} \rho / d T^{2} \sim t^{-3 / 2}$ sufficiently close to $T_{c}$, as opposed to $d^{2} \rho / d T^{2} \sim t^{-1}$ for $\xi(T) \ll \ell$ 【.

Our reasoning goes as follows. The adequate description of electric transport on scales larger than the phasebreaking length $L_{\phi}$ is a network of resistors, made up of 


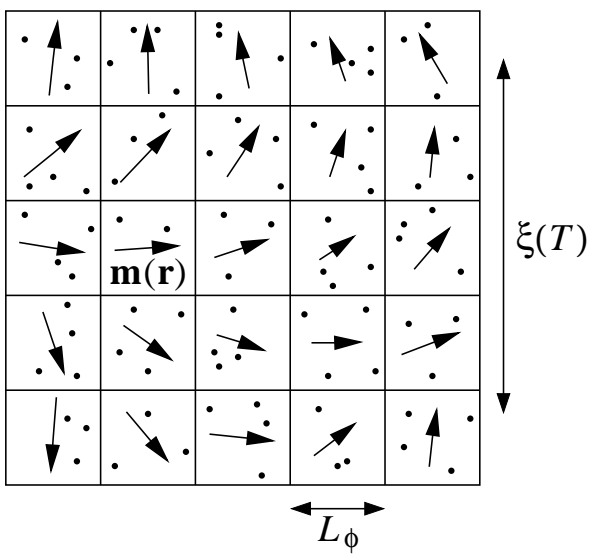

FIG. 1: Schematic representation of resistor network describing disordered ferromagnets when the correlation length $\xi(T)$ exceeds the phase-coherence length $L_{\phi}$. Each block of size $L_{\phi}$ constitutes a resistor with coarse-grained magnetization (arrows) and random impurities (dots).

cubes of size $L_{\phi}$, as illustrated in Fig. 1 This network is inhomogeneous due to the different spin and disorder configurations in each cube. Contrary to the Boltzmann prescription, it is essential to first compute the effective resistivity of the inhomogeneous network and to perform the disorder and thermal averages only as the last step of the calculation. Then, correlations between distant spins affect the resistance through the inhomogeneous current and field distribution over the network, leading to a true singularity for $t \rightarrow 0$ even within MF theory.

To illustrate the importance of performing the impurity and thermal averages at the last step of the calculation, consider a minimal model of two macroscopic resistors in sequence. These resistors differ in both impurity and spin configurations. Within the Boltzmann approach, both resistances are equal to $1 / \bar{\sigma}$ upon configurational and thermal averaging, yielding a total resistance of $2 / \bar{\sigma}$. However, for the actual distribution of impurities and spins, their conductivities differ, so that $\sigma_{1}=\bar{\sigma}+\delta \sigma / 2$ while $\sigma_{2}=\bar{\sigma}-\delta \sigma / 2$. Then, the effective resistance becomes $\left(\sigma_{1}+\sigma_{2}\right) / \sigma_{1} \sigma_{2} \simeq 2 / \bar{\sigma}+\delta \sigma^{2} / 2 \bar{\sigma}^{3}$ (assuming $\delta \sigma \ll \bar{\sigma}$ ). This involves an additional term $\delta \sigma^{2} / 2 \bar{\sigma}^{3}$ with non-zero average.

Effective conductivity of an inhomogeneous medium.Remarkably, the effective resistivity $\rho_{\text {eff }}=1 / \sigma_{\text {eff }}$ can be computed for an arbitrary realization $\sigma(\mathbf{r})$ of the local conductivity, provided that the relative variation in $\sigma(\mathbf{r})$ is weak 11]. To see this, we decompose the current, the conductivity, and the electric field into averages $\mathbf{j}_{0}$, $\sigma_{0}$, and $\mathbf{E}_{0}$ and spatially fluctuating contributions $\delta \mathbf{j}(\mathbf{r})$, $\delta \sigma(\mathbf{r})$, and $\delta \mathbf{E}(\mathbf{r})$. From Ohm's law, we have

$$
\begin{aligned}
\mathbf{j}_{0} & =\sigma_{0} \mathbf{E}_{0}+\langle\delta \sigma(\mathbf{r}) \delta \mathbf{E}(\mathbf{r})\rangle, \\
\delta \mathbf{j}(\mathbf{r}) & =\delta \sigma(\mathbf{r}) \mathbf{E}_{0}+\sigma_{0} \delta \mathbf{E}(\mathbf{r}) .
\end{aligned}
$$

Here, the brackets denote a spatial average. Combining the continuity equation $\boldsymbol{\nabla} \cdot \delta \mathbf{j}=0$ and Maxwell's equation $\boldsymbol{\nabla} \times \delta \mathbf{E}=0$ with Eq. (3), one obtains $\delta \mathbf{E}(\mathbf{q})=$ $-\hat{\mathbf{q}} \hat{\mathbf{q}} \cdot \mathbf{E} \delta \sigma(\mathbf{q}) / \sigma_{0}$, where $\hat{\mathbf{q}}$ denotes the unit vector in the direction of the wave vector q. Inserting this into Eq. (21), one finds for the effective macroscopic conductivity $\sigma_{\text {eff }}$ (defined by $\mathbf{j}_{0}=\sigma_{\text {eff }} \mathbf{E}_{0}$ ) in three dimensions

$$
\sigma_{\text {eff }}=\sigma_{0}-\frac{\left\langle[\delta \sigma(\mathbf{r})]^{2}\right\rangle}{3 \sigma_{0}} .
$$

Remarkably, this result is independent of the geometry of the conductivity variations. To illustrate this, consider a sample with a 50-50 random mixture of domains of conductivities $\sigma_{1}$ and $\sigma_{2}$, where $\left|\sigma_{1}-\sigma_{2}\right| \ll \sigma_{1}, \sigma_{2}$. In this case, Eq. (44) yields $\sigma_{\text {eff }}=\sigma_{0}-\left(\sigma_{1}-\sigma_{2}\right)^{2} / 12 \sigma_{0}$, which is completely independent of the arrangement of domains. It is interesting to remark that in two dimensions, one can find an exact, geometry-independent expression for such two-phase systems which is valid for arbitrarily large inhomogeneities $\left|\sigma_{2}-\sigma_{1}\right| /\left(\sigma_{1}+\sigma_{2}\right)[12$.

Effective conductivity due to spin fluctuations.-The conductance of a phase-coherent sample exhibits random, sample-specific, and reproducible variations as a function of external parameters such as the Fermi energy $E_{F}$. These conductance fluctuations arise due to interference between different elastic scattering paths of a carrier that diffuses through the sample [13]. Thus, the conductance $g\left(\mathbf{r}, E_{F}\right)$ varies from block to block because of their different impurity configurations. The fluctuations in the conductivity entering Eq. (4) can then be expressed as

$$
\delta \sigma(\mathbf{r})=\delta g\left(\mathbf{r}, E_{F} ; \mathbf{m}(\mathbf{r})\right) / L_{\phi} .
$$

We assume that the system is so large that domains with any magnetization combined with any disorder realization appear. Using this ergodicity assumption we replace the spatial average in Eq. (4) by independent disorder and thermal (magnetization) averages.

Two spin subbands. - In our case, the external parameter is a vector, namely the magnetization $\mathbf{m}(\mathbf{r})$. To proceed, we first consider the simplest case in which the dominant effect of the impurity spins on the carriers is an effective Zeeman field arising from the exchange interaction, which is proportional to the magnetization $m(\mathbf{r})$. Then the coarse-grained magnetization $\mathbf{m}(\mathbf{r})$, which is averaged over each cube of size $L_{\phi}$, can be incorporated via equal, but opposite energy shifts $\pm \alpha m(\mathbf{r})$ for spin-up and spin-down carriers, and $\delta \sigma$ becomes a sum of contributions from the two spin projections,

$$
\begin{aligned}
\delta \sigma(\mathbf{r}) & =\left[\delta g_{\uparrow}\left(\mathbf{r}, E_{F}\right)+\delta g_{\downarrow}\left(\mathbf{r}, E_{F}\right)\right] / L_{\phi} \\
& =\left[\delta g\left(\mathbf{r}, E_{F}^{+}(\mathbf{r})\right)+\delta g\left(\mathbf{r}, E_{F}^{-}(\mathbf{r})\right)\right] / L_{\phi} .
\end{aligned}
$$

Here, $g\left(\mathbf{r}, E_{F}\right)$ is the spinless conductance of the cube at position $\mathbf{r}$ and we have introduced the shifted Fermi energies $E_{F}^{ \pm}=E_{F} \pm \alpha m$, see Fig. 20 We used that carriers 


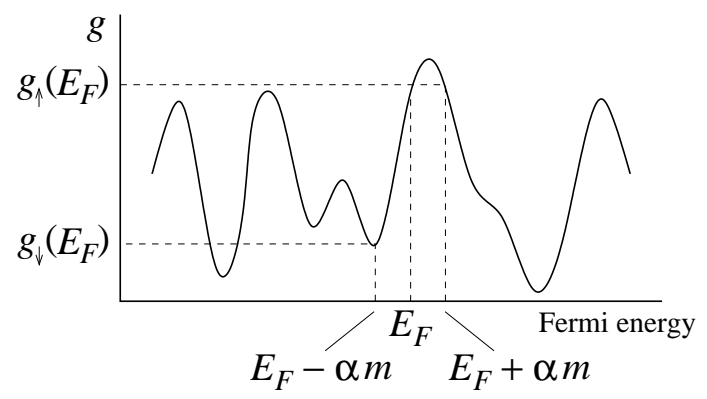

FIG. 2: Sample-specific variation of $g\left(E_{F}\right)$ for a block of the resistor network (UCF) in the absence of spin. The conductances for each spin direction are obtained by including equal, but opposite exchange-induced Zeeman shifts of the Fermi energy. As indicated, this leads to a difference in the conductances for the two spin directions.

with both spin projections are scattered from the same impurities. Substituting Eq. (6) into Eq. (4), we obtain

$$
\begin{aligned}
\rho_{\text {eff }}-\rho_{0} & =\frac{2 \rho_{0}^{3}}{3 L_{\phi}^{2}}\left\{\left\langle\left[\delta g\left(\mathbf{r}, E_{F}^{+}(\mathbf{r})\right)\right]^{2}\right\rangle+\left\langle\left[\delta g\left(\mathbf{r}, E_{F}^{-}(\mathbf{r})\right)\right]^{2}\right\rangle\right. \\
+ & \left.\left\langle\delta g\left(\mathbf{r}, E_{F}+\alpha m(\mathbf{r})\right) \delta g\left(\mathbf{r}, E_{F}-\alpha m(\mathbf{r})\right)\right\rangle\right\} \cdot(7)
\end{aligned}
$$

The first two terms on the rhs describe universal conductance fluctuations, which are independent of the Fermi energy and thus of $m(\mathbf{r})[13]$. Therefore, the only $t$ dependence comes from the conductance correlator.

It is well known 13. that this conductance correlator is a function $F(x)$ of the dimensionless ratio $x=\alpha m / E_{c}$, where $E_{c}=D / L_{\phi}^{2}$ is the inverse diffusion time through a block of size $L_{\phi}$ ( $D$ denotes the diffusion constant). In three dimensions, the asymptotic behaviors of $F(x)$ are given by 13. $F(x)=F(0)\left(1-C_{1} x^{2}\right)$ for $x \ll 1$ and $F(x)=F(0) C_{2} x^{-1 / 2}$ for $x \gg 1$, where $C_{1}$ and $C_{2}$ are constants of order unity. The remaining step is to substitute $F(x)$ into Eq. (7) and to perform the thermodynamic average over $\mathbf{m}$, using the MF distribution for $t>0$,

$$
\mathcal{P}[\mathbf{m}(\mathbf{r})] \propto \exp \left\{-\frac{c}{2 T} \int d^{3} r\left[a^{2} \nabla_{i} \mathbf{m} \cdot \nabla_{i} \mathbf{m}+t \mathbf{m}^{2}\right]\right\} .
$$

Here, $c a^{2}$ is a spin stiffness. Using this distribution, one finds for the magnetization fluctuations

$$
\left\langle\mathbf{m}^{2}(\mathbf{r})\right\rangle=\int_{q<1 / L_{\phi}} \frac{d^{3} q}{(2 \pi)^{3}} \frac{3 T_{c} / c}{t+q^{2} a^{2}} .
$$

The restriction in the range of the $\mathbf{q}$ integration accounts for the fact that we are computing fluctuations of the coarse-grained magnetization. The appearance of $t$ in the denominator of Eq. (9) manifests the fact that the net strength of fluctuations grows when approaching $T_{c}$. This is in contrast to the conventional origin of the $t$-dependence, namely the spin correlations. Performing the integration in Eq. (9), we obtain $\left\langle\mathbf{m}^{2}(\mathbf{r})\right\rangle=$ $\left(3 T_{c} / 2 \pi^{2} c a^{2}\right)\left[1 / L_{\phi}-(1 / \xi(T)) \arctan \left(\xi(T) / L_{\phi}\right)\right]$

in terms of the MF correlation length $\xi(T)=a / \sqrt{t}$.

As the next step, we replace $m(\mathbf{r})$ in Eq. (7) by $\left\langle\mathbf{m}^{2}(\mathbf{r})\right\rangle^{1 / 2}$. Using the distribution (8), this procedure can be shown to be exact in the limits of small and large $x$. Expanding the result in the small parameter $L_{\phi} / \xi(T)=L_{\phi} \sqrt{t} / a$, we readily obtain

$$
\rho_{\mathrm{eff}}-\rho_{0}=\frac{2 \rho_{0}^{3}}{3 L_{\phi}^{2}}\left[F\left(x_{0}\right)-F^{\prime}\left(x_{0}\right) x_{0} \frac{\pi L_{\phi} \sqrt{t}}{4 a}\right],
$$

where $x_{0}=\left(\alpha / E_{c}\right)\left(3 T_{c} / 2 \pi^{2} c a^{2} L_{\phi}\right)^{1 / 2}$. The $t$-dependent part of $\rho_{\text {eff }}$ is given by the second term on the rhs 14.

So far, our model completely disregards spin-orbit (SO) coupling which is present in the vast majority of ferromagnets. Below, we incorporate SO coupling into the calculation of the effective resistivity.

Spin-orbit coupling.--In the presence of SO coupling, the variance $\left\langle\left[\delta g\left(\mathbf{r}, E_{F} ; \mathbf{m}(\mathbf{r})\right)\right]^{2}\right\rangle_{\text {imp }}$ increases by a factor of two when applying a sufficiently strong Zeeman field [15]. In terms of the relevant dimensionless measure of the exchange-induced Zeeman field $y=$ $\alpha m \tau_{\text {so }}$ (here $\tau_{\text {so }}$ is the SO time), we can then write $\left\langle\left[\delta g\left(\mathbf{r}, E_{F} ; \mathbf{m}(\mathbf{r})\right)\right]^{2}\right\rangle_{\text {imp }}=H(y)$ with $H(\infty) / H(0)=2$ [15]. Combining Eqs. (5) and (4), substituting $H(y)$, and proceeding as in the derivation of Eq. (10), we obtain 14]

$$
\rho_{\mathrm{eff}}-\rho_{0}=\frac{2 \rho_{0}^{3}}{3 L_{\phi}^{2}}\left[H\left(y_{0}\right)-H^{\prime}\left(y_{0}\right) y_{0} \frac{\pi L_{\phi} \sqrt{t}}{4 a}\right],
$$

where $y_{0}=\alpha \tau_{\mathrm{so}}\left(3 T_{c} / 2 \pi^{2} c a^{2} L_{\phi}\right)^{1 / 2}$. Eq. (11) assumes that the SO length $\ell_{\mathrm{so}}=\left(D \tau_{\mathrm{so}}\right)^{1 / 2}$ is smaller than $L_{\phi}$.

Discussion.-By going beyond the Boltzmann description in calculating the critical behavior of the resistivity, we have expressed $\rho_{\text {eff }}$ through mesoscopic characteristics. Recall that our results, Eqs. (10) and (11), were obtained within MF theory so that the singularity in the $t$-dependence of $\rho_{0}$ on the lhs of these equations, arising within the Boltzmann formalism, is suppressed by impurities, since $\xi(T) \gg \ell$. Eqs. (10) and (11) show that in addition to this suppression, impurity scattering leads to a $\sqrt{t}$ singularity which is much stronger than the de Gennes-Friedel result $t \ln (1 / t)$ and the largewave-vector Fisher-Langer contribution. This singularity, which in essence is governed by Kirchhoff's laws, constitutes our central result. To resolve the anomaly on top of a monotonous phonon contribution to $\rho$, it is customary to consider $d^{2} \rho / d T^{2}$. Then, our disorder-induced MF anomaly becomes $d^{2} \rho / d T^{2} \sim t^{-3 / 2}$. In a log-log plot, the slope is -1 in the Boltzmann regime $\xi(T) \ll \ell$ and $-3 / 2$ for the disorder-induced anomaly, as shown in Fig. 3

According to Eqs. (10) and (11), the sign of the anomaly is governed by the sign of either $F^{\prime}\left(x_{0}\right)$ or $H^{\prime}\left(y_{0}\right)$, depending on the strength of SO coupling. Since $F^{\prime}\left(x_{0}\right)<0$ while $H^{\prime}\left(y_{0}\right)>0$, the disorder-induced anomaly corresponds to a decrease of $d^{2} \rho / d T^{2}$ when approaching $T_{c}$ from above in the absence of SO coupling 


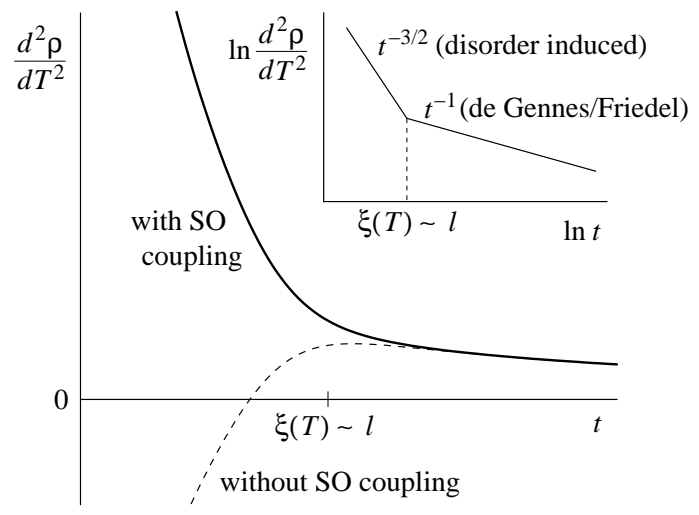

FIG. 3: Resistive anomaly within MF theory for $L_{\phi} \sim \ell$ (schematic) with (full line) and without (dashed line) SO coupling. The anomaly is described by the de Gennes-Friedel mechanism for $\xi(T) \ll \ell$, while the disorder-induced mechanism of this paper dominates closer to $T_{c}$ where $\xi(T) \gg L_{\phi}$. When $L_{\phi} \gg \ell$, there is an additional intermediate regime. Inset: anomaly with SO coupling in a log-log plot.

and to an increase in the realistic case of strong SO coupling. The difference in signs comes about because the Zeeman field suppresses the correlator in Eq. (7), while it increases the UCF for strong SO interactions.

The magnitude of the disorder-induced anomaly is controlled by $F(0)$ and $H(0)$, which are the variances of the conductance, $\left\langle(\delta g)^{2}\right\rangle_{\mathrm{imp}}$, cf. Eqs. (10) and (11). This quantity assumes different values in different regimes which are defined by the relations between the relevant lengths, namely $L_{\phi}$, the thermal length $L_{T}=$ $(D / T)^{1 / 2}$, and the spin-flip length $\ell_{s}$. In the simplest case, when $L_{\phi}$ is the smallest length, $L_{\phi} \ll L_{T}, \ell_{s}$, we have $\left\langle(\delta g)^{2}\right\rangle_{\text {imp }} \sim\left(e^{2} / h\right)^{2}$ [13]. If $L_{\phi}$ is larger than $L_{T}$ or $\ell_{s}$, then $\left\langle(\delta g)^{2}\right\rangle_{\text {imp }}$ is suppressed below the universal limit, $\left\langle(\delta g)^{2}\right\rangle_{\mathrm{imp}} \sim\left(e^{2} / h\right)^{2}\left[\min \left\{L_{T}, \ell_{s}\right\} / L_{\phi}\right][13]$.

The disorder-induced anomaly proposed in this paper is most relevant to ferromagnets with high resistance and low $T_{c}$ since in such systems (i) the mean free path is dominated by impurity scattering $[16$ and (ii) the phasecoherence length exceeds the mean free path at $T_{c}$. Natural candidates for disordered low- $T_{c}$ ferromagnets are $\mathrm{SrRuO}_{3}$ 17] which belongs to the class of poor metals 18 as well as diluted magnetic semiconductors (DMS) 19, 20] which lately attracted considerable attention in view of possible spintronics applications 21]. Indeed, both types of materials show resistive anomalies which differ significantly from the predictions of Fisher-Langer theory. Metallic samples of DMS exhibit pronounced maxima near $T_{c}$ even in $\rho$ vs. $T$ 22, 23, 24, 25, 26, 27, 28], which are unrelated to the $T=0$ metal-insulator transition [24, 29].

In closing, it is interesting to point out that our principal result, namely the enhancement of the resistive anomaly by disorder, can be viewed in perspective of the enhanced coupling of the spin fluctuations to the carriers, expected due to the diffusive carrier dynamics.

Two of us (MER and FvO) acknowledge the hospitality of the Weizmann Institute (supported by the Einstein Center and LSF grant HPRI-CT-2001-00114) where this work was initiated, as well as of the MPI for Complex Systems. This work was also supported by NSF-DAAD Grant No. INT-0231010 and Sfb 290.

[1] W. Gerlach, Physik. Z. 33, 953 (1932); H. Bittel and W. Gerlach, Ann. Physik 33, 661 (1938).

[2] P. P. Craig et al., Phys. Rev. Lett. 19, 1334 (1967).

[3] See e.g. H. Yanagihara et al., Phys. Rev. B 65, 092411 (2002) and references therein.

[4] P. G. de Gennes and J. Friedel, J. Phys. Chem. Solids 4, 71 (1958).

[5] M. E. Fisher and J. S. Langer, Phys. Rev. Lett. 20, 665 (1968).

[6] D. J. W. Geldart and T. G. Richard, Phys. Rev. B 12, 5175 (1975); D. J. W. Geldart, Phys. Rev. B 15, 3455 (1977).

[7] O. Entin-Wohlman, G. Deutscher, and R. Orbach, Phys. Rev. B 11, 219 (1975).

[8] D. J. W. Geldart and K. De'Bell, Phys. Rev. B 32, 3285 (1985).

[9] I. Schuller, R. Orbach, and P. M. Chaikin, Phys. Rev. Lett. 41, 1413 (1978).

[10] M. Kataoka, Phys. Rev. B 63, 134435 (2001).

[11] See L. D. Landau and E. M. Lifshitz, Electrodynamics of Continuous Media (Pergamon Press, Oxford, 1984) for a related calculation of the effective dielectric constant.

[12] A. M. Dykhne, Zh. Eksp. Fiz. 59, 110 (1970) [Sov. Phys. JETP 32, 63 (1971)].

[13] A. D. Stone, Phys. Rev. Lett. 54, 2692 (1985); B. L. Altshuler, Pis'ma Zh. Eksp. Teor. Fiz. 51, 530 (1985); P. A. Lee and A. D. Stone, Phys. Rev. Lett. 55, 1622 (1985); P. A. Lee, A. D. Stone, and H. Fukuyama, Phys. Rev. B 35, 1039 (1987).

[14] Going beyond Gaussian fluctuations, the square roots in Eqs. (10) and (11) change to $t^{\nu(1+\eta)}$, where $\nu(1+\eta) \approx$ 0.72 for the three-dimensional Heisenberg model.

[15] C. W. J. Beenakker, Rev. Mod. Phys. 69, 731 (1997).

[16] For $\ell_{\text {imp }} \gg \ell$, the disorder-induced anomaly is reduced by a reduction in the $\mathrm{UCF},\left\langle(\delta g)^{2}\right\rangle_{\mathrm{imp}} \sim\left(e^{2} / h\right)^{2}\left(\ell / \ell_{\mathrm{imp}}\right)$.

[17] L. Klein et al., Phys. Rev. Lett. 77, 2774 (1996).

[18] V. J. Emery and S. A. Kivelson, Phys. Rev. B 74, 3253 (1995).

[19] C. Timm, J. Phys.: Condens. Matter 15, R1865 (2003).

[20] Earlier work on the resistivity of DMS near $T_{c}$ does not go beyond Fisher-Langer theory or ignores critical fluctuations alltogether, see M. P. López-Sancho and L. Brey, Phys. Rev. B 68, 113201 (2003) and references therein.

[21] See e.g., I. Žutić, J. Fabian, and S. Das Sarma, Rev. Mod. Phys. 76, 323 (2004).

[22] A. Van Esch et al., Phys. Rev. B 56, 13103 (1997).

[23] F. Matsukura et al., Phys. Rev. B 57, R2037 (1998).

[24] H. Ohno et al., J. Appl. Phys. 85, 4277 (1999).

[25] T. Hayashi et al., Appl. Phys. Lett. 78, 1691 (2001).

[26] S. J. Potashnik et al., Appl. Phys. Lett. 79, 1495 (2001); 
M. B. Stone et al., Appl. Phys. Lett. 83, 4568 (2003).

[27] K. W. Edmonds et al., Appl. Phys. Lett. 81, 3010 (2002).

[28] T. Wojtowicz et al., Appl. Phys. Lett. 83, 4220 (2003).

[29] A detailed analysis should take into account the small value of $k_{F} a \ll 1$ in DMS. According to Ref. [4], this implies a wide resistivity peak with $\delta \rho \sim \rho$. The anomaly discussed here sits on top of this wide maximum in the immediate vicinity of $T_{c}$. 